\title{
柔性航天器快速机动过程主动振动与姿态控制 方法研究
}

\author{
董超, 徐世杰 ${ }^{*}$, 王浩, 张明星, 薛普 \\ 北京航天长征飞行器研究所, 北京 100076 \\ *联系人, E-mail: xushijiehit@126.com
}

收稿日期: 2021-05-10; 接受日期: 2021-08-30; 网络出版日期: 2021-09-16

\begin{abstract}
摘要 针对柔性航天器挠性附件振动与姿态机动耦合降低控制精度的问题, 给出了一种混合分力合成主动振动 控制方法, 给出了若干定理并进行了理论证明. 其次, 系统参数波动及随机干扰因素不可忽略, 对经典滑模控制器 进行优化, 形成一种新型自适应一滑模变结构控制器. 为应对控制力矩抖振和系统参数鲁棒性差等缺点, 引入新型 滑模边界层和力矩更新率. 最后，提出了将混合分力合成方法与姿态控制相结合的策略. 借助压电智能材料实现 两者的联合应用。仿真结果表明, 改进的滑模变结构控制器可有效降低参数变动及外干扰影响, 结合混合分力合 成方法后，柔性附件残余振动得到了明显的控制，进一步提高了姿态精度.
\end{abstract}

关键词柔性航天器, 混和分力合成方法, 主动振动-自适应滑模变结构控制

PACS: $45.05 .+\mathrm{x}, 45.82 .+\mathrm{r}, 46.40 .-\mathrm{f}$

\section{1 概述}

随着航天技术的发展，当前柔性航天器附件趋向 大型化、轻质化，此类低阻尼柔性结构刚柔耦合特性 明显 ${ }^{[1]}$. 在姿态机动、变轨等空间任务执行过程中, 容 易产生残余振动且难以迅速衰减, 另外, 柔性航天器系 统参数不确定性和外干扰影响不可忽视，这都将对航 天器系统稳定性和姿态精度造成明显的影响.

近年来众多学者对柔性航天器的主动振动控制和 姿态控制方法开展了大量研究. 在柔性附件主动振动 控制方面，分力合成主动振动控制方法以其简单、有 效的突出优势受到广泛关注. 分力合成方法的核心思
想是按照一定规则将合力拆分成分力并进行重新组 合, 使各分力引起的系统振动最终相互抵消, 达到振动 控制的目的. 该方法由Liu等人 ${ }^{[2]}$ 最先于 1988 年提出, 随后将该方法应用到了绕单轴转动的柔性系统姿态控 制中, 获得了较好的振动控制效果. 蔡晓雷 ${ }^{[3]}$ 以空间交 会对接任务为背景，用分力合成方法对任务中柔性附 件的振动问题进行了有效控制. 考虑到柔性系统存在 参数变动的情况，陕晋军等人 ${ }^{[4,5]}$ 给出了分力合成方法 对参数变动鲁棒性提升的改进和证明. 随后多位学者 对分力合成方法的应用做了拓展性研究 ${ }^{[6-9]}$, 虽然分力 合成方法对柔性附件振动控制效果明显，但受到开环 控制特性的影响，难以直接应用到航天器高精度姿态

引用格式: 董超, 徐世杰, 王浩, 等. 柔性航天器快速机动过程主动振动与姿态控制方法研究. 中国科学: 物理学 力学 天文学, 2021, 51: 104704 Dong C, Xu S J, Wang H, et al. Flexible spacecraft attitude maneuver via adaptive sliding mode control and mixed component synthesis vibration suppression (in Chinese). Sci Sin-Phys Mech Astron, 2021, 51: 104704, doi: 10.1360/SSPMA-2021-0116 
控制中.

针对现代柔性航天器对快速、高精度姿态控制的 需求，自适应控制 ${ }^{[10-12]}$ 、滑模变结构控制 ${ }^{[13,14]}$ 、最优 控制 ${ }^{[15,16]}$ 、非线性控制及复合控制等方法近来得到了 广泛的应用 ${ }^{[17,18]}$. 其中, 滑模变结构控制方法是一种优 势较明显的鲁棒性控制方法，众多学者对其在姿态机 动控制方面的应用进行了大量探索. Maganti和 $\mathrm{Singh}^{[19]}$ 提出可将飞行器俯仰角及其一阶导数看作输 出变量, 通过最小系统相位推导得出新型俯仰角控制 率, 确保系统参数稳定. Chen和Huang ${ }^{[20]}$ 针对受连续扰 动航天器因残余振动明显进而影响姿态精度的问题, 探索了将鲁棒控制与自适应方法相结合的可行性. Zhu等人 ${ }^{[21]}$ 重点针对参数波动对姿态精度影响明显的 问题，提出以不同滑模控制器分别实现扰动估计和姿 态控制. 虽然滑模变结构控制器在姿态机动控制方面 具备明显优势，但仍然存在输出控制力矩颤振及无法 同时对柔性附件残余振动抑制的问题. 智能压电材料 技术的发展，为主动振动控制方法的应用提供了新的 解决途径 ${ }^{[22-26]}$.

综上所述, 基于当前研究现状和实际需求, 针对当 前面临的实际问题, 本文主要工作如下.

(1) 针对由系统参数不确定性和外部干扰引起的 输出力矩颤振问题，提出一种改进的自适应滑模变结 构控制(Adaptive Sliding Mode Variable Structure Controller, ASMVSC)策略. 降低了输出力矩对参数准确性 的依赖, 提高了对外部干扰的鲁棒性.

(2) 为抑制柔性附件残余振动, 提出混合分力合成 (Mixd Component Synthesis Vibration Suppression, MCSVS)方法. 该振动控制策略与其他现有策略的最 大不同在于，在智能压电材料的辅助下能够同时完成 姿态控制和对柔性附件残余振动的抑制.

本文第2节给出了柔性航天器的动力学模型, 第3 节介绍了MCSVS方法和主动振动控制器，第4节中给 出了改进的ASMVSC及其稳定性证明。第5节通过仿 真算例验证了提出的控制策略的有效性. 最后给出文 章结论.

\section{2 系统动力学建模}

柔性航天器一般可以看作簇状系统，太阳能帆 板、柔性天线等为主要的柔性附件. 柔性航天器主要
包括中心刚体和连接在中心刚体上的柔性附件. 带柔 性附件的航天器可以简化成为如图 1所示的二维示 意图.

图1中，灰色方框表示中心刚体. 椭圆虚线表示柔 性附件发生柔性变形前的状态，椭圆实线表示柔性附 件经柔性变形后的状态. 在惯性坐标系 $O X Y Z$ 中对模型 进行矢量描述, 不失一般性, 假设柔性附件上有一质量 微元 $\mathrm{d} m, \mathbf{R}$ 表示从惯性坐标系原点至柔性附件上任意 质量微元 $\mathrm{d} m$ 的矢径, $\mathbf{w}$ 表示柔性附件上质量微元 $\mathrm{d} m$ 的 柔性变形量. 采用牛顿-欧拉方法对柔性航天器进行动 力学建模, 引入四元数描述航天器姿态. 柔性航天器动 力学模型可表示为式(1) ${ }^{[27]}$ :

$$
\left\{\begin{array}{l}
{\left[\begin{array}{c}
\dot{q}_{0} \\
\dot{\mathbf{q}}
\end{array}\right]=\frac{1}{2}\left[\begin{array}{c}
-\mathbf{q}^{\mathrm{T}} \\
\left(q_{0} \mathbf{I}_{3}+\mathbf{q}^{\times}\right)
\end{array}\right] \boldsymbol{\omega},} \\
\mathbf{J} \dot{\boldsymbol{\omega}}+\boldsymbol{\delta}^{\mathrm{T}} \ddot{\boldsymbol{\eta}}+\boldsymbol{\omega}^{\times}\left(\mathbf{J} \boldsymbol{\omega}+\boldsymbol{\delta}^{\mathrm{T}} \dot{\boldsymbol{\eta}}\right)=\mathbf{T}+\mathbf{u}, \\
\boldsymbol{\delta} \dot{\boldsymbol{\omega}}+\ddot{\boldsymbol{\eta}}+\mathbf{C} \dot{\boldsymbol{\eta}}+\mathbf{K} \boldsymbol{\eta}=-\boldsymbol{\delta}_{\mathrm{p}} u_{\mathrm{p}},
\end{array}\right.
$$

式中, $\mathbf{J}$ 为系统不考虑柔性附件相对中心刚体转动的惯 量矩阵; $\boldsymbol{\omega}$ 为系统姿态角速度; $\boldsymbol{\delta}$ 为柔性附件振动与系 统姿态运动的耦合矩阵; $\boldsymbol{\eta}$ 为模态坐标向量; $\mathbf{C}$ 和 $\mathrm{K}$ 分 别为系统阻尼阵与刚度阵; $\boldsymbol{\delta}_{\mathrm{p}}$ 为压电智能材料电压输 入与柔性附件振动耦合矩阵; $\mathbf{T}$ 为控制力矩.

\section{3 混合分力合成主动振动控制方法}

\section{1 混合分力合成原理}

不失一般性，以式(2)所示的有阻尼二阶振动系统 为例

$\ddot{x}+2 \zeta \omega_{n} \dot{x}+\omega_{n}^{2} x=F(t)$.

首先给出两个关于混合分力合成的定理:

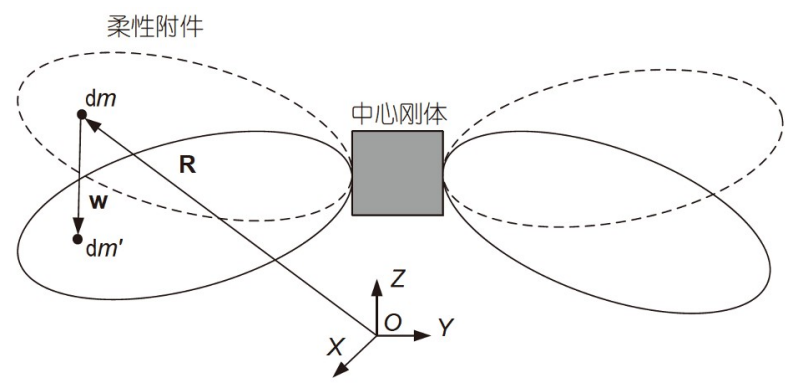

图 1 簇状柔性航天器模型示意图

Figure 1 Clustered flexible spacecraft model. 
定理1 对有阻尼二阶振动系统, 零初始状态下, 若 将振动的半周期划分成均等的 $n$ 份 ( $n$ 为奇数), 在每份 起点或与起点延后整倍周期处，依次作用正负值顺序 变更的 $n$ 个形式相似的分力, 则当合成力作用结束时, 系统无残余振动.

定理 2 对有阻尼二阶振动系统, 零初始状态下, 若 将振动周期划分成均等的 $n$ 份 ( $n$ 为偶数), 在每份起点 或与起点延后整倍周期处，依次作用正负值顺序变更 的 $n$ 个形式相似的分力, 则当合成力作用结束时, 系统 无残余振动.

证明：由于混合分力合成方法中分力的方向有正 有负, 因此, 按照 $n$ 的奇偶性, 各分力的作用时间为

$\tau_{i}= \begin{cases}(i-1) \frac{1}{n} \frac{\pi}{\omega_{d}}+q_{i} \frac{2 \pi}{\omega_{d}}, n \text { 为奇数, } \\ (i-1) \frac{1}{n} \frac{2 \pi}{\omega_{d}}+q_{i} \frac{2 \pi}{\omega_{d}}, n \text { 为偶数, }\end{cases}$

其中 $i=1,2, \ldots ; q_{i}=0,1, \ldots$.

作用力可表示为

$$
F(t)=\sum_{i=1}^{n}(-1)^{i+1} \mathrm{e}^{-\zeta \omega_{n} \tau_{i}} f\left(t-\tau_{i}\right) u\left(t-\tau_{i}\right) .
$$

\section{系统解析解为}

$$
\begin{aligned}
X(t)= & \frac{1}{\omega_{d}} \sum_{i=1}^{n} \int_{0}^{t}(-1)^{i+1} \mathrm{e}^{-\zeta \omega_{n} \tau_{i}} f\left(\xi-\tau_{i}\right) \\
& \times \mathrm{e}^{-\zeta \omega_{n}(t-\xi)} \sin \left[\omega_{d}(t-\xi)\right] \mathrm{d} \xi .
\end{aligned}
$$

将每个分力按半周期划分成 $k$ 段，则式(5)可描 述为

$$
\begin{aligned}
X(t)= & \frac{1}{\omega_{d}} \sum_{i=1}^{n} \sum_{j=1}^{k} \int_{\tau_{i}+(j-1) \frac{\pi}{\omega_{d}}}^{\tau_{i}+j \frac{\pi}{\omega_{d}}}(-1)^{i+1} \mathrm{e}^{-\zeta \omega_{n} \tau_{i}} \\
& \times f\left(\xi-\tau_{i}\right) \mathrm{e}^{-\zeta \omega_{n}(t-\zeta)} \sin \left[\omega_{d}(t-\xi)\right] \mathrm{d} \xi .
\end{aligned}
$$

对式中变量做变量代换:

$$
\eta_{i}=\xi-\tau_{i}-(j-1) \frac{\pi}{\omega_{d}} \text {. }
$$

经变量代换后, 系统响应可表述为

$$
\begin{aligned}
X(t)= & \frac{1}{\omega_{d}} \sum_{i=1}^{n} \sum_{j=1}^{k} \int_{0}^{\frac{\pi}{\omega_{d}}}(-1)^{i+1} \\
& \left.\times \mathrm{e}^{-\zeta \omega_{n} \tau_{i} f}\left(\eta_{i}+(j-1) \frac{\pi}{\omega_{d}}\right) \mathrm{e}^{-\zeta \omega_{n}\left(t-\eta_{i}-\tau_{i}-(j-1) \frac{\pi}{\omega_{d}}\right.}\right) \\
& \times \sin \left[\omega_{d}\left(t-\eta_{i}-(j-1) \frac{\pi}{\omega_{d}}-\tau_{i}\right)\right] \mathrm{d} \eta_{i} .
\end{aligned}
$$

积分运算只受积分计算上下限的影响，因此，对 式(8)中 $\eta_{i}$ 下标忽略并设

$$
\alpha=\omega_{d}\left[t-\eta-(j-1) \frac{\pi}{\omega_{d}}\right] .
$$

最终, 系统响应解析解可表述为

$$
\begin{aligned}
X(t)= & \frac{1}{\omega_{d}} \sum_{j=1}^{k} \int_{0}^{\frac{\pi}{\omega_{d}}} f\left(\eta+(j-1) \frac{\pi}{\omega_{d}}\right) \\
& \times \mathrm{e}^{-\zeta \omega_{n}}\left(t-\eta-(j-1) \frac{\pi}{\omega_{d}}\right) \\
& \times \sum_{i=1}^{n}(-1)^{i+1} \sin \left(\alpha-\omega_{d} \tau_{i}\right) \mathrm{d} \eta .
\end{aligned}
$$

当 $n$ 取奇数时, 系统响应最后求和项可以进一步简 化为

$$
\begin{aligned}
\sum_{i=1}^{n}( & -1)^{i+1} \sin \left(\alpha-\frac{(i-1) \pi}{n}\right) \\
= & \sum_{g=0}^{(n-1) / 2} \sin \left(\alpha-\frac{2 g \pi}{n}\right) \\
& +\sum_{g=1}^{(n-1) / 2} \sin \left(\alpha-\frac{(2 g-1+n) \pi}{n}\right) .
\end{aligned}
$$

考虑关系式:

$$
\left\{\begin{array}{l}
\sum_{i=1}^{n} \sin \left[\alpha-\frac{(i-1) 2 \pi}{n}\right]=0, \\
\sum_{i=1}^{n} \cos \left[\alpha-\frac{2(i-1) \pi}{n}\right]=0 .
\end{array}\right.
$$

则求和项为

$$
\begin{aligned}
\sum_{i=1}^{n}(-1)^{i+1} \sin \left(\alpha-\frac{(i-1) \pi}{n}\right) \\
=\sum_{i=1}^{n} \sin \left(\alpha-\frac{2(i-1) \pi}{n}\right)=0 .
\end{aligned}
$$

因此, 当 $n$ 取奇数时, 所有分力结束后, 系统响应为 0 . 证毕.

当 $n$ 取偶数时, 式(9)可以表示为

$$
\begin{aligned}
X(t)= & \frac{1}{\omega_{d}} \sum_{j=1}^{k} \int_{0}^{\frac{\pi}{\omega_{d}}} f\left(\eta+(j-1) \frac{\pi}{\omega_{d}}\right) \\
& \times \mathrm{e}^{-\zeta \omega_{n}}\left(t-\eta-(j-1) \frac{\pi}{\omega_{d}}\right) \\
& \times \sum_{i=1}^{n}(-1)^{i+1} \sin \left(\alpha-\frac{2(i-1) \pi}{n}\right) \mathrm{d} \eta .
\end{aligned}
$$

考虑关系式: 


$$
\left\{\begin{array}{l}
\sin (y)=\frac{\mathrm{e}^{\mathrm{j} y}-\mathrm{e}^{-\mathrm{i} y}}{2 \mathrm{j}}, \\
\cos (y)=\frac{\mathrm{e}^{\mathrm{j} y}-\mathrm{e}^{-\mathrm{j} y}}{2},
\end{array}\right.
$$

式中, $j=\sqrt{-1}$ 为复数单位, 式(14)中最后的求和项可进 一步展开为

$$
\begin{aligned}
& \sum_{i=1}^{n}(-1)^{i+1} \sin \left(\alpha-\frac{2(i-1) \pi}{n}\right) \\
& =\frac{1}{2 \mathrm{j}}\left[\mathrm{e}^{\mathrm{j} \alpha} \frac{1-\mathrm{e}^{-2 \pi \mathrm{j}}}{\left.1-\mathrm{e}^{-4 \pi \mathrm{j} / n}-\mathrm{e}^{-\mathrm{j} \alpha} \frac{1-\mathrm{e}^{2 \pi \mathrm{j}}}{1-\mathrm{e}^{4 \pi \mathrm{j} / n}}\right]}\right. \\
& -\frac{1}{2 \mathrm{j}}\left[\begin{array}{l}
\mathrm{e}^{\mathrm{j} \alpha} \frac{\mathrm{e}^{-2 \pi \mathrm{j} / n}\left(1-\mathrm{e}^{-2 \pi \mathrm{j}}\right)}{1-\mathrm{e}^{-4 \pi \mathrm{j} / n}} \\
\left.-\mathrm{e}^{-\mathrm{j} \alpha} \frac{\mathrm{e}^{2 \pi \mathrm{j} / n}\left(1-\mathrm{e}^{2 \pi \mathrm{j}}\right)}{1-\mathrm{e}^{4 \pi \mathrm{j} / n}}\right] .
\end{array}\right.
\end{aligned}
$$

考虑到:

$$
\left\{\begin{array}{l}
\mathrm{e}^{-2 \pi \mathrm{j}}=\cos (-2 \pi)+\mathrm{j} \sin (-2 \pi)=1, \\
\mathrm{e}^{2 \pi \mathrm{j}}=\cos (2 \pi)+\mathrm{j} \sin (2 \pi)=1 .
\end{array}\right.
$$

可得, 式(14)最终结果同样为 0 .

至此，混和分力合成方法对有阻尼系统的应用有 效性得以完成证明. 为体现分力合成方法最基本原理, 考虑令 $n=2$ 及 $n=3$, 各分力、合力及相应响应随时间变 化如图2-5所示.

\section{2 混和分力合成主动振动控制器}

为了便于展示控制器的控制效果, 这里取 $n=2$. 针 对式(1)中第3式表示的振动方程，以第一阶振动为对

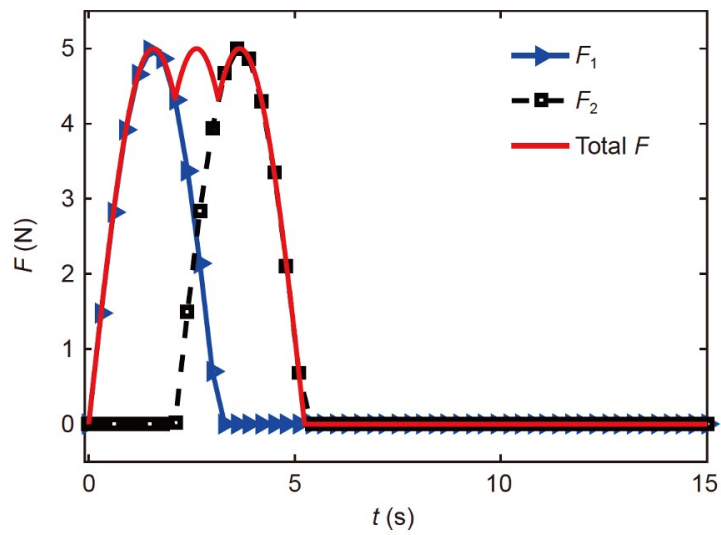

图 2 (网络版彩图) $n=2$ 时, 合力和分力随时间变化示意图

Figure 2 (Color online) Time-varying components and synthesis at $n=2$.

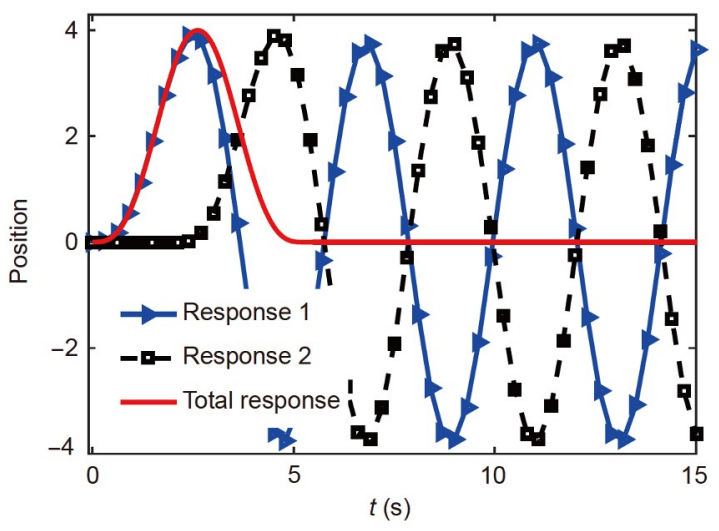

图 3 (网络版彩图) $n=2$ 时, 合力及各分力对应的系统响应 示意图

Figure 3 (Color online) Response of components and synthesis at $n=2$.

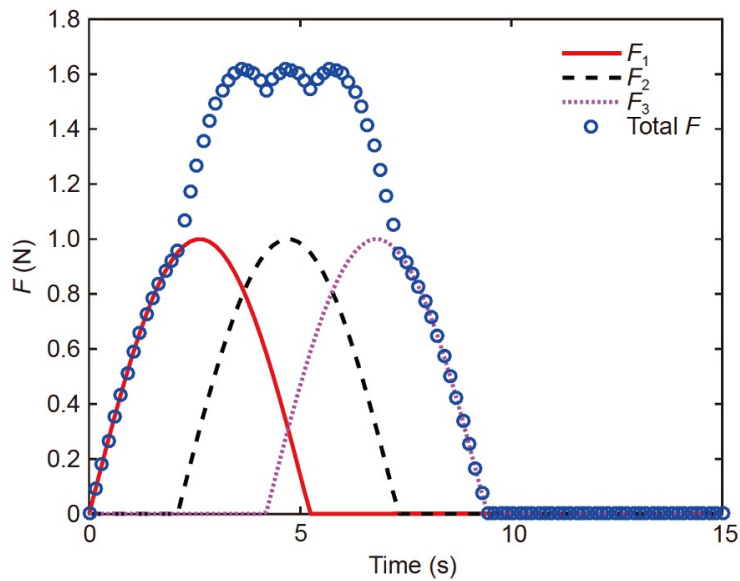

图 4 (网络版彩图) $n=3$ 时, 合力和分力随时间变化示意图

Figure 4 (Color online) Time-varying components and synthesis at $n=3$.

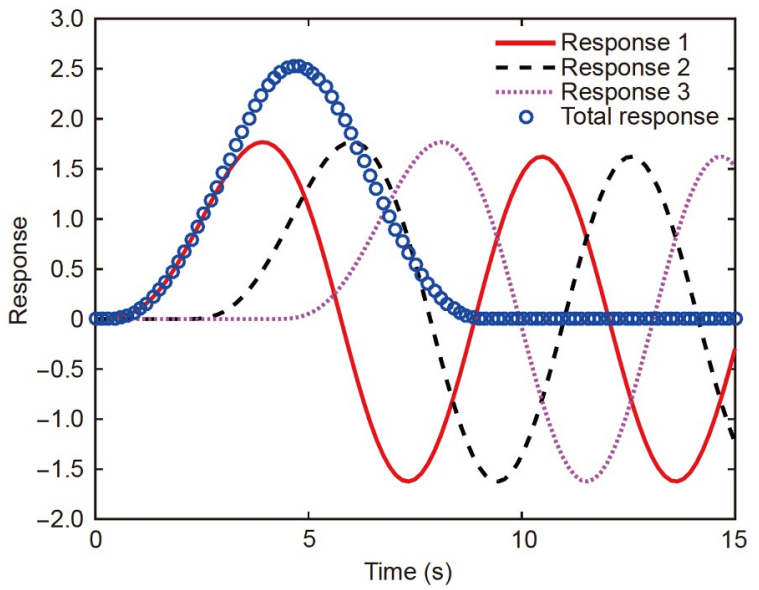

图 5 (网络版彩图) $n=3$ 时，合力及各分力对应的系统响应 示意图

Figure 5 (Color online) Response of components and synthesis at $n=3$. 
象, 令其右侧遵循分力合成规则:

$$
-\left(\boldsymbol{\delta}_{\mathrm{p}} u_{\mathrm{p}}\right)_{i}-(\boldsymbol{\delta} \dot{\boldsymbol{\omega}})_{i}=F=F_{1}+F_{2} .
$$

考虑到第 $i$ 阶振动阻尼系数的影响, 有

$$
F_{2}=\mathrm{e}^{-\frac{\zeta_{i} \pi}{\sqrt{1-\zeta_{i}^{2}}}} F_{1} \mathrm{e}^{-s t_{2}}
$$

式中, $t_{2}=\frac{\pi}{\omega \sqrt{1-\zeta_{i}^{2}}}$.

因此, 各分力的取值应满足如下规则:

$$
F_{1}(t)+F_{2}\left(t+t_{2}\right)=-(\boldsymbol{\delta} \dot{\boldsymbol{\omega}}) .
$$

使压电智能材料的电压输入值满足式(18)和(20) 的要求, 则将能够实现对系统柔性附件残余振动的主 动抑制.

\section{4 改进的自适应滑模变结构控制器设计}

本节将给出一种改进的自适应滑模变结构控制 器, 并对闭环系统的稳定性给出证明.

设定有如下形式表示的转换函数:

$\mathbf{S}(\mathbf{x})=\left[\begin{array}{ll}\mathbf{I}_{3 \times 3} & \mathbf{F}_{3 \times 3}\end{array}\right] \mathbf{x}$,

式中, $\mathbf{F}_{3 \times 3}$ 为正定的对称矩阵. 为更好地适应实际工程 需求，将自适应控制率与滑模变结构控制方法相结合， 给出如下形式所示的自适应滑模变结构控制器:

$\mathbf{u}=-\mathbf{P S}-\frac{\varepsilon \mathbf{S}}{\|\mathbf{S}\|_{2}+\tau}$,

式中, $\mathbf{P}$ 和 $\tau$ 为控制器的参数, 参数 $\varepsilon$ 满足以下规则:

$\varepsilon=\widehat{\kappa}_{0}+\widehat{\kappa}_{1}\|\mathbf{q}\|_{2}+\widehat{\kappa}_{2}\|\boldsymbol{\omega}\|_{2}$,

$\dot{\hat{\kappa}_{0}}=p_{0}\|\mathbf{S}\|_{2}$,

$\dot{\widehat{\kappa}}_{1}=p_{1}\|\mathbf{S}\|_{2} \cdot\|\mathbf{q}\|_{2}$,

$\dot{\widehat{\kappa}}_{2}=p_{2}\|\mathbf{S}\|_{2} \cdot\|\boldsymbol{\omega}\|_{2}$,

式中, $p_{i}>0(i=0,1,2)$, 参数取值情况与系统实际状态 有关.

定理3 针对式(1)所表示的柔性航天器动力学模 型, 在考虑参数不确定性和有外干扰的情况下, 式(22) 所示的自适应控制器能使系统快速完成期望的姿态机 动任务.
证明: 构造Lyapunove函数:

$$
V=\frac{1}{2}\left(\mathbf{S}^{\mathrm{T}} \mathbf{J S}+\frac{\kappa_{0 \delta}^{2}}{p_{0}}+\frac{\kappa_{1 \delta}^{2}}{p_{1}}+\frac{\kappa_{2 \delta}^{2}}{p_{2}}\right),
$$

式中, $\kappa_{i \delta}=\kappa_{i}-\widehat{\kappa}_{i}$.

与转换函数式(21)相结合, 整理可得

$$
\begin{aligned}
\dot{V}= & \mathbf{S}^{\mathrm{T}}\left((\mathbf{F q}-\mathbf{S})^{\times} \mathbf{J} \boldsymbol{\omega}+\frac{1}{2} \mathbf{F J}\left(q_{0} \mathbf{I}_{3}+\mathbf{q}^{\times}\right) \boldsymbol{\omega}\right) \\
& +\mathbf{S}^{\mathrm{T}} \mathbf{d}+\mathbf{S}^{\mathrm{T}} \mathbf{u}-\sum_{i=0}^{2} \frac{\kappa_{i \delta} \dot{\hat{\kappa}}_{i}}{p_{i}} .
\end{aligned}
$$

考虑到 $\mathbf{S}^{\mathrm{T}} \mathbf{S}^{\times}=0$, 于是, 式(28)可进一步简化:

$$
\begin{aligned}
\dot{V}= & \mathbf{S}^{\mathrm{T}} \mathbf{F}\left(\mathbf{q}^{\times} \mathbf{J} \boldsymbol{\omega}+\frac{1}{2} \mathbf{F J}\left(q_{0} \mathbf{I}_{3}+\mathbf{q}^{\times}\right) \boldsymbol{\omega}\right) \\
& +\mathbf{S}^{\mathrm{T}} \mathbf{d}+\mathbf{S}^{\mathrm{T}} \mathbf{u}-\sum_{i=0}^{2} \frac{\kappa_{i \delta} \dot{\hat{\kappa}_{i}}}{p_{i}} \\
\leq & \left(\left\|\boldsymbol{q}^{\times}\right\|_{2}+\frac{1}{2}\left\|q_{0} \mathbf{I}_{3}+\mathbf{q}^{\times}\right\|_{2}\right) \cdot\|\mathbf{J}\|_{2} \cdot\|\mathbf{S}\|_{2} \cdot\|\boldsymbol{\omega}\|_{2} \cdot\|\mathbf{F}\|_{2} \\
& +\mathbf{S}^{\mathrm{T}} \mathbf{d}+\mathbf{S}^{\mathrm{T}} \mathbf{u}-\sum_{i=0}^{2} \frac{\kappa_{i \delta} \frac{\dot{\hat{\kappa}_{i}}}{p_{i}} .}{}
\end{aligned}
$$

依据向量二范数性质, 四元数具有如下特点:

$\left\{\begin{array}{l}\|\mathbf{q}\| \leq 1, \\ \left\|\mathbf{q}^{\times}+q_{0} \mathbf{I}_{3}\right\| \leq 1 .\end{array}\right.$

将式(30)代入式(29)有

$\dot{V} \leq\|\mathbf{S}\|_{2}\left(\kappa_{0}+\kappa_{1}\|\mathbf{q}\|_{2}+\kappa_{2}\|\boldsymbol{\omega}\|\right)+\mathbf{S}^{\mathrm{T}} \mathbf{u}-\sum_{i=0}^{2} \frac{\kappa_{i \delta} \dot{\widehat{\kappa}}_{i}}{p_{i}}$.

将未连续化的自适应滑模变结构控制器表达式代 入上式并进行整理, 可得

$$
\begin{aligned}
\dot{V} \leq & \mathbf{S}^{\mathrm{T}}(-\mathbf{P S})-\varepsilon \mathbf{S}^{\mathrm{T}} \frac{\mathbf{S}}{\|\mathbf{S}\|_{2}} \\
& +\|\mathbf{S}\|_{2}\left(\kappa_{0}+\kappa_{1}\|\mathbf{q}\|_{2}+\kappa_{2}\|\boldsymbol{\omega}\|_{2}\right) \\
& -\kappa_{0 \delta}\|\mathbf{S}\|_{2}-\kappa_{1 \delta}\|\mathbf{S}\|_{2} \cdot\|\mathbf{q}\|_{2}-\kappa_{2 \delta}\|\mathbf{S}\|_{2} \cdot\|\boldsymbol{\omega}\|_{2} \\
= & \mathbf{S}^{\mathrm{T}}(-\mathbf{P S})-\|\mathbf{S}\|_{2}\left(\kappa_{0}+\kappa_{1}\|\mathbf{q}\|_{2}+\kappa_{2}\|\boldsymbol{\omega}\|_{2}\right) \\
& +\|\mathbf{S}\|_{2}\left(\kappa_{0}+\kappa_{1}\|\mathbf{q}\|_{2}+\kappa_{2}\|\boldsymbol{\omega}\|_{2}\right)-\kappa_{0 \delta}\|\mathbf{S}\|_{2} \\
& -\kappa_{1 \delta}\|\mathbf{S}\|_{2} \cdot\|\mathbf{q}\|_{2}-\kappa_{2 \delta}\|\mathbf{S}\|_{2} \cdot\|\boldsymbol{\omega}\|_{2} \\
= & -\mathbf{S}^{\mathrm{T}} \mathbf{P S} .
\end{aligned}
$$

由于 $\mathbf{P}$ 为正定对称矩阵, 因此可得 $\dot{V} \leq 0$, 其稳定性 证毕. 
系统在滑模面 $\mathbf{S}(\mathbf{x})=\mathbf{0}$ 上将保持稳定状态.

证明: 根据前文可得

$0<V(t) \leq V(0)<\infty$.

根据积分关系式 $\int_{0}^{t} \dot{V}(\alpha) \mathrm{d} \alpha=V(t)-V(0)$, 可得

$-\int_{0}^{t} \dot{V}(\alpha) \mathrm{d} \alpha \leq V(0)$.

将式(32)代入式(34), 经整理可得

$\lim _{t \rightarrow+\infty} \int_{0}^{t} \mathbf{S}^{\mathrm{T}} \mathbf{P S} \mathrm{d} \alpha \leq \lim _{t \rightarrow+\infty} V(0)$.

结合Barbalat引理内容, 可得

$\lim _{t \rightarrow+\infty} \mathbf{S}=\mathbf{0}$.

得证.

通过对系统状态变量和转换函数的整合, 可得

$$
\boldsymbol{\omega}=-\mathbf{F q} \text {. }
$$

将式(37)代入式(1)第1式，引入参数矩阵 $\mathbf{F}=\operatorname{diag}[f$, $f, f]$, 可得

$$
\left\{\begin{array}{l}
\dot{\mathbf{q}}=-\frac{1}{2} f\left(q_{0} \mathbf{I}_{3}+\mathbf{q}^{\times}\right) \mathbf{q}=-\frac{1}{2} q_{0} f \mathbf{q}, \\
\dot{q}_{0}=-\frac{1}{2} \mathbf{q}^{\mathrm{T}}(-f \mathbf{q})=\frac{1}{2} f\left(1-q_{0}^{2}\right) .
\end{array}\right.
$$

设定 $q_{0}=q_{0}(t), t_{\mathrm{s}}$ 为动点到达切换平面的时刻，对 式(38)第2式求积分:

$$
\begin{aligned}
& q_{0}(t)=1-\frac{2\left(1-q_{0}\left(t_{\mathrm{s}}\right)\right) \mathrm{e}^{-f\left(t-t_{\mathrm{s}}\right)}}{1+q_{0}\left(t_{\mathrm{s}}\right)+\left(1-q_{0}\left(t_{\mathrm{s}}\right)\right) \mathrm{e}^{-f\left(t-t_{\mathrm{s}}\right)}}, t \geq t_{\mathrm{s}} . \\
& \quad \text { 显然, 当 } \lim _{t \rightarrow+\infty} q_{0}=1 \text { 时, 系统将到达平衡点 } \mathbf{x}=\mathbf{0} .
\end{aligned}
$$

\section{5 算例分析}

为验证本文所提出控制方法的有效性，本节给出 典型柔性航天器在不同条件、不同控制器下姿态机动 及柔性附件残余振动的分析对比.

\section{1 参考控制器控制效果分析}

式(40)和(41)分别给出了考虑和不考虑参数不确 定性的转动惯量矩阵:

$$
\begin{aligned}
\mathbf{J} & =\left[\begin{array}{ccc}
355.46 & -20.28 & -0.1933 \\
-20.28 & 210.3467 & -9.1567 \\
-0.1933 & -9.1567 & 266.39
\end{array}\right]\left(\mathrm{kg} \mathrm{m}^{2}\right), \\
\mathbf{J} & =\left[\begin{array}{ccc}
355.46 \times \text { rand } & -20.28 & -0.1933 \\
-20.28 & 210.3467 \times \text { rand } & -9.1567 \\
-0.1933 & -9.1567 & 266.39 \times \text { rand }
\end{array}\right]
\end{aligned}
$$

$$
\left(\mathrm{kg} \mathrm{m}^{2}\right),(0.9 \leq \text { rand } \leq 1.1) .
$$

系统固有频率为 $\boldsymbol{\omega}=\left[\begin{array}{llll}0.7681 & 1.1038 & 1.8733 & 2.5496\end{array}\right](\mathrm{rad} / \mathrm{s})$. 阻尼系数为 $\zeta=\left[\begin{array}{llll}5.607 & 8.62 & 12.83 & 25.16\end{array}\right] \times 10^{-3}$. 系统耦合 矩阵为

$$
\boldsymbol{\delta}=\left[\begin{array}{ccc}
6.45637 & 1.27814 & 2.15629 \\
-1.25619 & 0.91756 & -1.67264 \\
1.1687 & 2.48901 & -0.83674 \\
1.23637 & -2.6581 & -1.12503
\end{array}\right]\left(\sqrt{\mathrm{kg}} \mathrm{m} / \mathrm{s}^{2}\right),
$$

$\boldsymbol{\delta}_{\mathrm{p}}=\left[\begin{array}{c}2.342552 \\ -0.4225368 \\ 3.912984 \\ 7.026176\end{array}\right] \times 10^{-2}\left(\sqrt{\mathrm{kg}} \mathrm{m} /\left(\mathrm{Vs}^{2}\right)\right)$.

外干扰力矩设定为

$$
\begin{aligned}
\mathbf{T}(t) & =\left[\begin{array}{c}
-3+4 \cos (0.2 \pi t)-\cos (0.4 \pi t)+2 \omega_{1} \sin (0.11 t) \\
4+3 \sin (0.2 \pi t)-2 \cos (0.4 \pi t)+\omega_{2} \cos (0.11 t) \\
-3+4 \sin (0.2 \pi t)-3 \sin (0.4 \pi t)+2 \omega_{3} \sin (0.11 t)
\end{array}\right] \\
& \times 10^{-3}(\mathrm{~N} \mathrm{~m}) .
\end{aligned}
$$

柔性航天器姿态要求从 $\mathbf{Q}_{0}=\left[\begin{array}{ll}0.25882 & -0.31339\end{array}\right.$

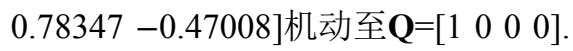

(1) 情况A: 标准控制器控制下不考虑参数不确定 性和外干扰力矩的系统姿态机动过程

有如下所示标准控制器:

$\mathbf{u}_{\mathrm{b}}=-\mathbf{P}_{\mathrm{b}} \mathbf{S}-\mathbf{H} \frac{\mu \mathbf{S}}{\|\mathbf{S}\|_{2}+\gamma}$,

式中, $\mathbf{P}_{\mathrm{b}}=\operatorname{diag}\left(\mathrm{P}_{\mathrm{b} 1}, \ldots, \mathrm{P}_{\mathrm{b} i}, \mathrm{P}_{\mathrm{b} i}>0\right), \mathbf{H}=\operatorname{diag}\left(H_{1}, \ldots, H_{i}\right.$, $\left.H_{i}>0\right), \mu$ 和 $\gamma$ 均为设定参数.

取 $\mathbf{P}_{\mathrm{b}}=\operatorname{diag}(15,15,15), \quad \mathbf{H}=\operatorname{diag}(1.5,1.5,1.5)$ ， $\mu=1, \gamma=3$.

图6给出了情况A下柔性航天器系统姿态机动过 程典型参数变化情况.

如图6(a)-(d)所示情况A下柔性航天器的姿态机动 情况. 图6(a)中, 四元数在 $40 \mathrm{~s}$ 左右到达期望状态. 在 图6(b)中, 滑模面数值下降迅速. 图6(c)给出了以 $5 \mathrm{~N} \mathrm{~m}$ 

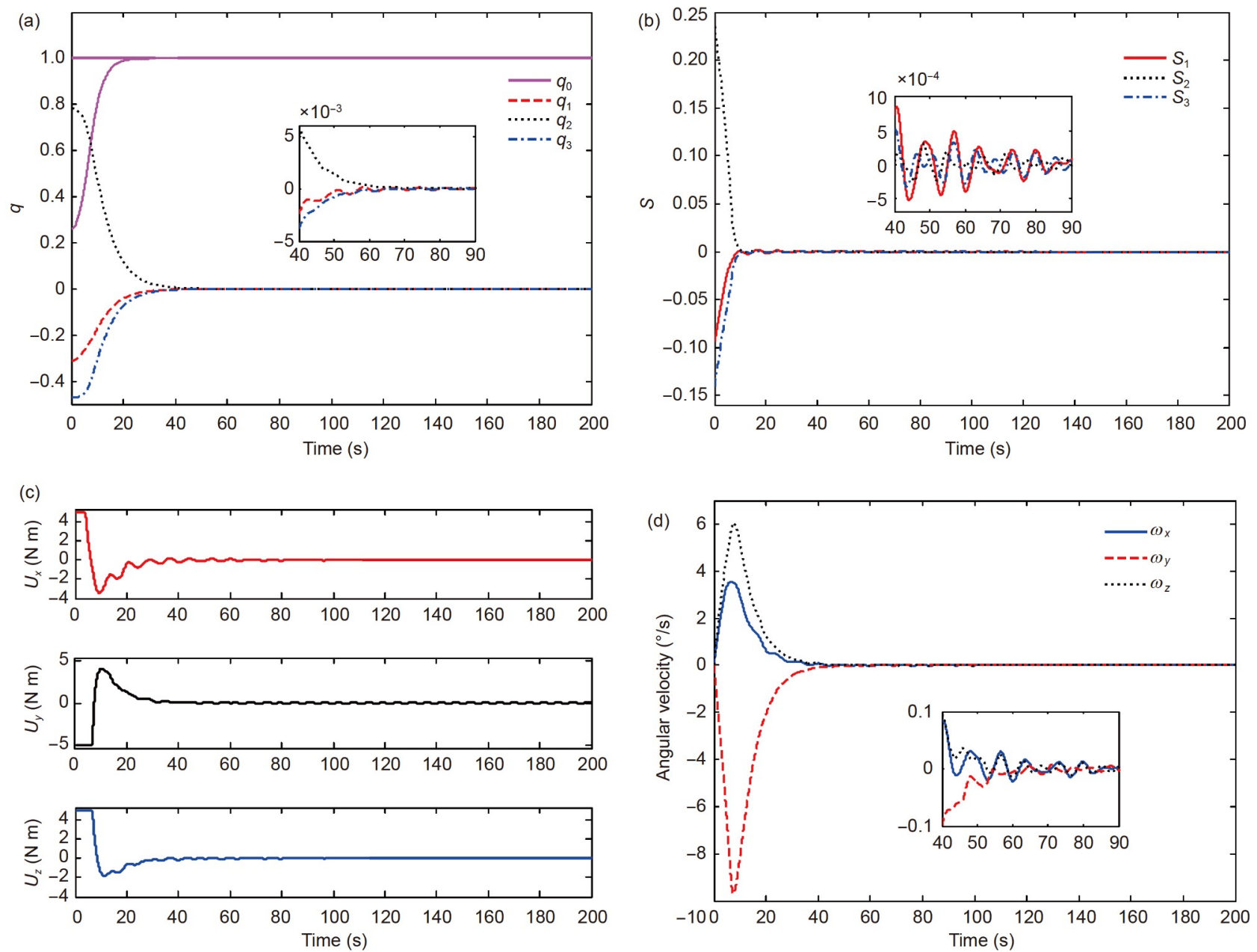

图 6 (网络版彩图)情况A仿真结果. (a) 四元数随时间变化情况; (b) 滑模面随时间变化情况; (c) 控制力矩随时间变化情况; (d) 角速度随时间变化情况

Figure 6 (Color online) The simulation results of case A. (a) Quaternion; (b) the results of sliding surface; (c) output control torques; (d) angular velocity.

为上下界的控制力矩输出情况. 图6(d)为柔性航天器 三轴角速度变化情况, 从角速度变化波动比较明显.

(2) 情况B: 标准控制器控制下考虑参数不确定性 和外干扰力矩的系统姿态机动过程

图7给出了情况B下柔性航天器系统姿态机动过 程典型参数变化情况.

从仿真结果来看, 相比于情况 $\mathrm{A}$ 中的结果, 情况 $\mathrm{B}$ 中参数收玫时间要更长, 需要在第 $50 \mathrm{~s}$ 左右系统才能 进入稳定状态，相比于情况 $\mathrm{A}$ 延迟了 $10 \mathrm{~s}$. 图7(c)中所 示的控制力矩在姿态机动开始阶段颤振现象非常明 显. 从对比结果可以看出, 当引入参数不确定性和外干 扰后，将对系统姿态机动过程中的精度产生不利的
影响.

\section{2 改进的滑模变结构控制器效果分析}

本节仿真验证中, 系统参数及初始条件选取与情况 $\mathrm{B}$ 中的条件相同. 控制器式(22)中, $\mathbf{P}=\operatorname{diag}(200,200$, 200), $\tau=0.5$, 其余参数为计算过程变量. 图 8 给出了在 改进的滑模变结构控制器作用下, 柔性航天器系统姿 态机动过程主要参数变化情况.

收敛时间方面，在改进滑模变结构控制器作用下， 收玫时间明显减少. 在 $40 \mathrm{~s}$ 之后, 四元数波动范围降至 $2 \times 10^{-3}$ 之内. 相比于同时间段内情况B中 $2 \times 10^{-2}$ 的范围, 幅值降低了 1 个数量级, 体现了更高效的收玫效率. 

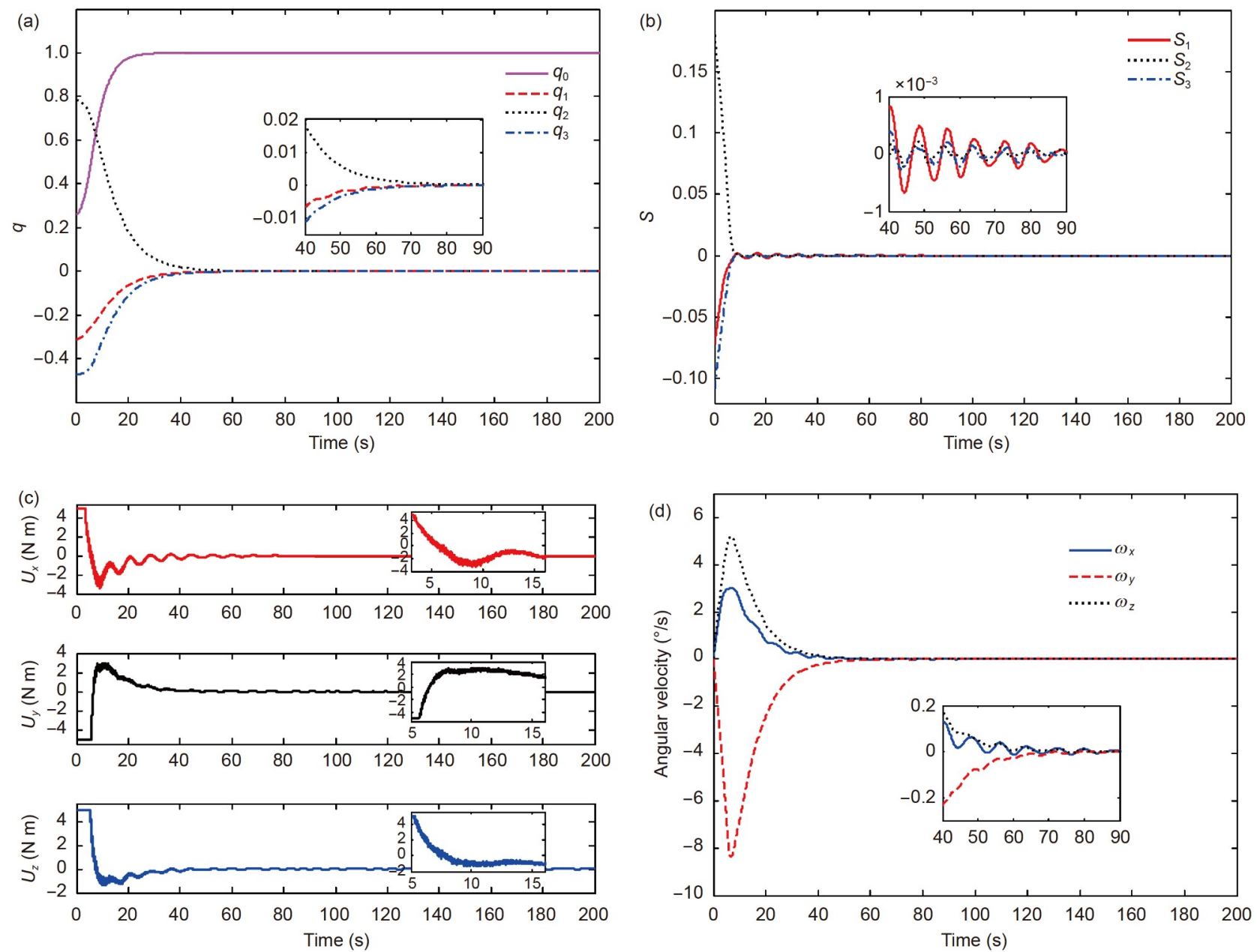

图 7 (网络版彩图)情况B仿真结果. (a) 四元数随时间变化情况; (b) 滑模面随时间变化情况; (c) 控制力矩随时间变化情况; (d) 角速度随时间变化情况

Figure 7 (Color online) The simulation results of case B. (a) Quaternion; (b) the results of sliding surface; (c) output control torques; (d) angular velocity.

图8(c)给出了控制力矩输出变化情况. 相比于情况B中 带有明显颤振现象的输出力矩, 经改进的滑模变结构 控制器优化后, 整个调姿过程输出力矩变化更加平稳. 图8(d)给出了角速度变化情况, 能够看出其达到稳定 状态的速度进一步提升. 通过仿真结果对比得出, 改 进的滑模变结构控制器能够更好地适应存在参数不确 定性和外干扰力矩情况的系统.

\section{3ＭCSVS主动振动控制方法效果分析}

为了保证对比效果，本节所选用的初始条件及参 数与前面小结中情况相同. 图9给出了在MCSVS控制 方法和改进的滑模变结构控制器同时控制作用下的仿
真分析结果.

同时考虑MCSVS方法和AMSVSC方法后, 四元数 收玫速度更快, 而且变化过程更加平滑, 进一步避免了 柔性附件姿态机动过程的残余振动累积. 从图9(b)滑 模面变化过程可以看出姿态机动过程中系统稳定性较 之前情况得到了明显提升. 图9(c)所示的控制输出力 矩颤振现象明显降低. 图9(d)给出了柔性航天器角速 度变化情况, 通过MCSVS方法和AMSVSC方法的联 合应用，柔性附件残余振动得到了明显抑制，使转动 角速度在姿态机动结束后的波动幅度进一步降低, 提 升柔性航天器系统的姿态精度. 分析结果表明, 结合 MCSVS和AMSVSC后, 系统稳定性明显提升. 

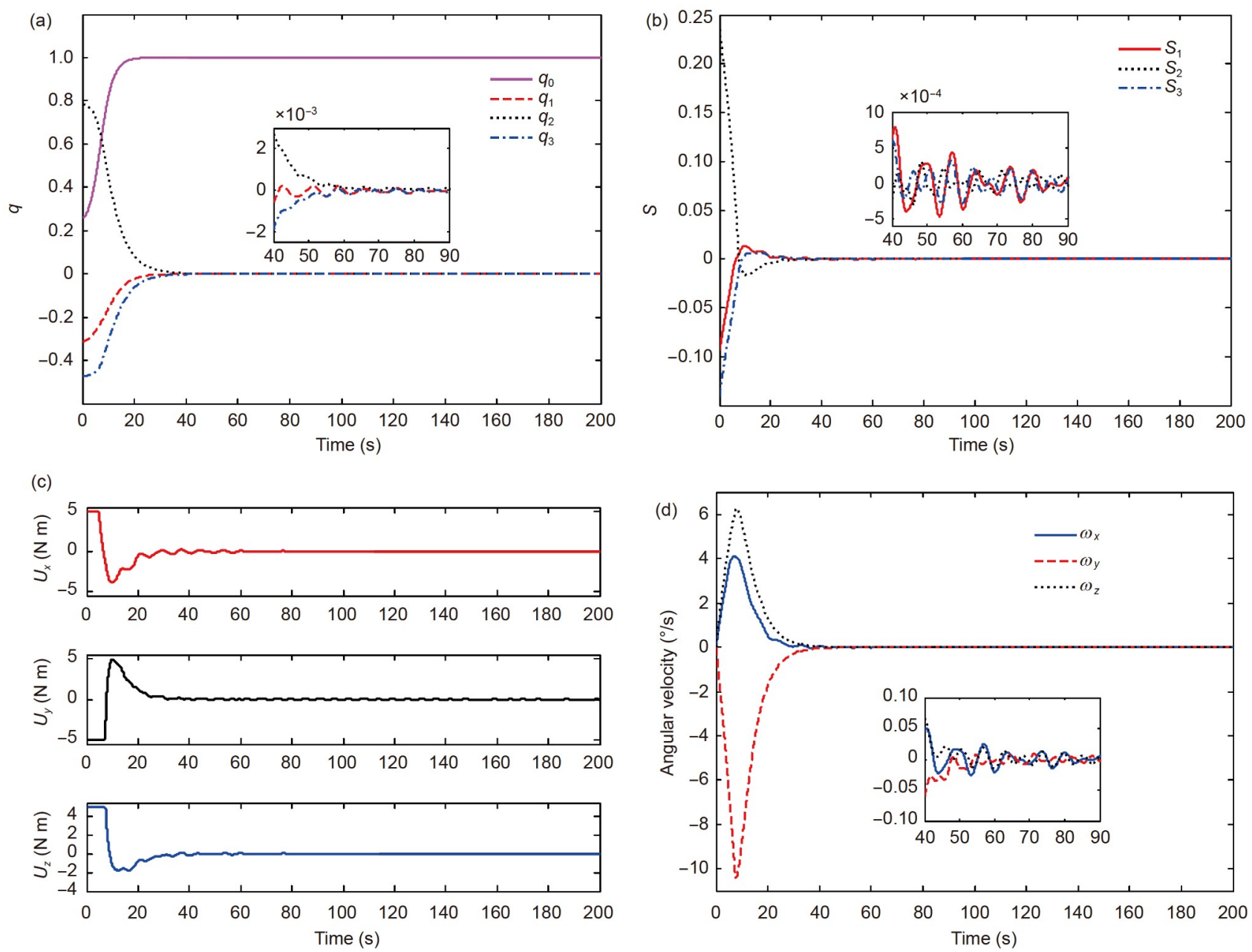

图 8 (网络版彩图)考虑改进的滑模变结构控制器的仿真结果. (a) 四元数随时间变化情况; (b) 滑模面随时间变化情况; (c) 控 制力矩随时间变化情况; (d) 角速度随时间变化情况

Figure 8 (Color online) The simulation results with AMSVSC. (a) Quaternion; (b) the results of sliding surface; (c) output control torques; (d) angular velocity.

为了更准确地评估MCSVS方法对柔性附件振动 的抑制情况，我们给出考虑和不考虑MCSVS方法下， 柔性附件的能量变化对比情况.

以模态坐标表示的柔性附件振动能量可表述为

$$
E=\dot{\boldsymbol{\eta}}^{\mathrm{T}} \dot{\boldsymbol{\eta}}+\boldsymbol{\eta}^{\mathrm{T}} \mathbf{K} \boldsymbol{\eta}
$$

考虑和不考虑MCSVS方法情况下，式(46)表示的 柔性附件能量变化如图10和11所示.

考虑MCSVS方法后，整个姿态机动过程中柔性附 件振动能量降低明显, 下降幅度大. 通过对比, MCSVS 方法对柔性附件振动抑制效果十分明显.

将上述仿真结果以表格形式进行整理，不同分析
条件下, 仿真分析结果如表1所示.

由以上分析及对比表格显示内容可见，本文所提 出的方法及MCSVS与AMSVSC联合应用策略对姿态 精度提升及残余振动控制具有明显效果.

\section{6 结论}

针对柔性航天器在参数不确定、外干扰条件下姿 态机动精度需进一步提升和柔性附件振动对姿态精度 影响明显的问题, 本文提出了一种将MCSVS方法和 AMSVSC方法相结合的控制策略, 能够实现在提升姿 态机动控制精度的同时实现对柔性附件振动的控制. 
(a)
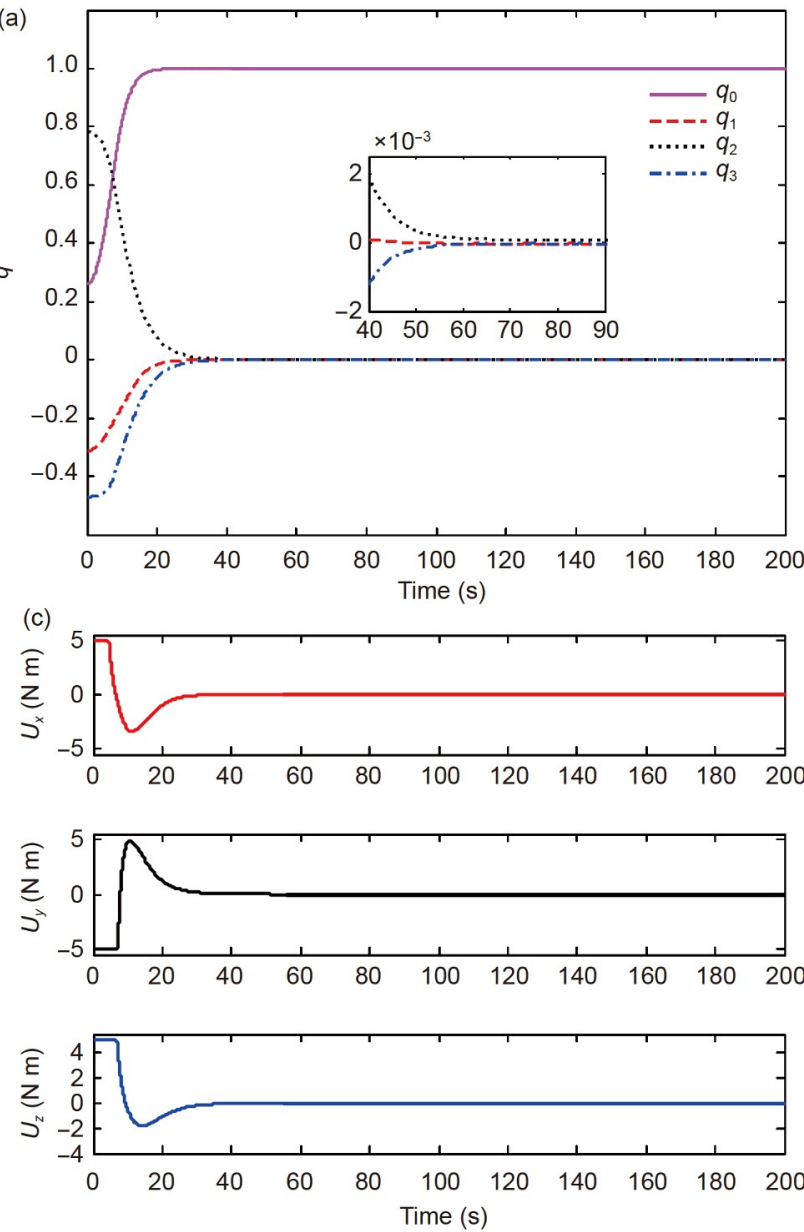

(b)
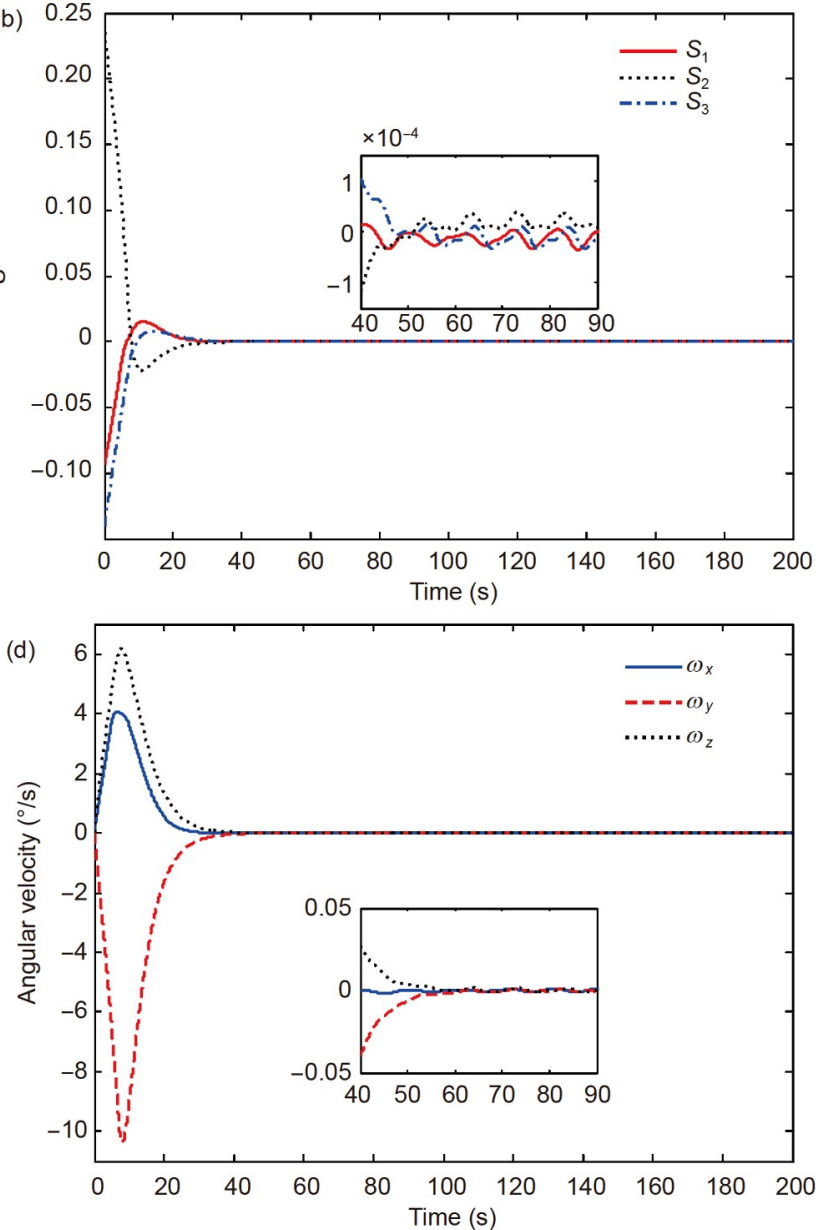

图 9 (网络版彩图)考虑混合分力合成和改进的滑模变结构控制器的仿真结果. (a) 四元数随时间变化情况; (b) 滑模面随时间 变化情况; (c) 控制力矩随时间变化情况; (d) 角速度随时间变化情况

Figure 9 (Color online) The simulation results with MCSVS and AMSVSC. (a) Quaternion; (b) the results of sliding surface; (c) output control torques; (d) angular velocity.

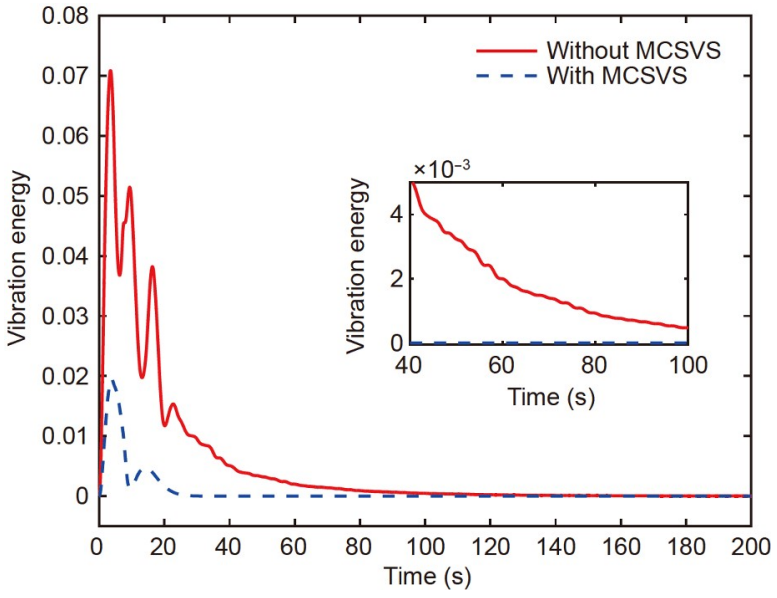

图 10 (网络版彩图)振动能量变化情况对比

Figure 10 (Color online) Vibration energy under AMSVSC with and without MCSVS.
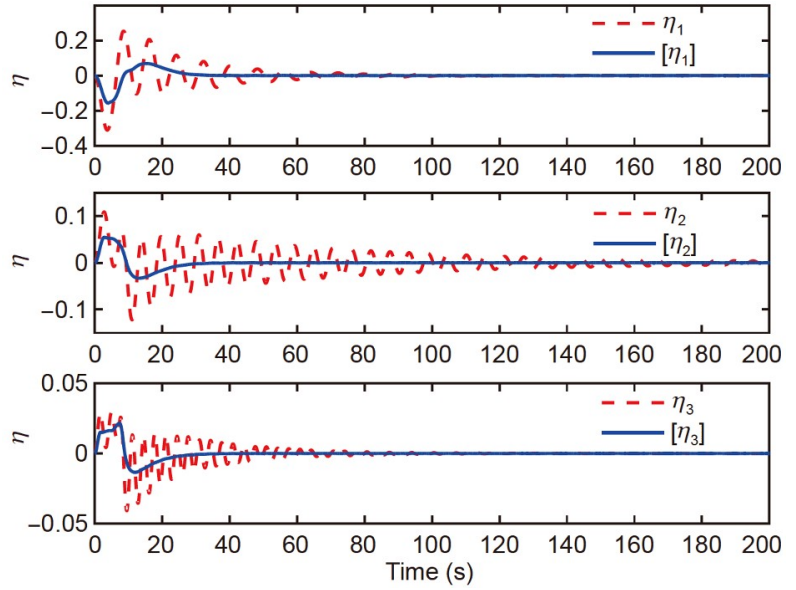

图 11 (网络版彩图)前三阶模态左边变化情况对比

Figure 11 (Color online) Mode displacement under AMSVSC with and without MCSVS. 
表 1 分析结果对比

Table 1 Comparison of simulation results

\begin{tabular}{|c|c|c|c|c|}
\hline 条件 & 四元数变化 & 滑模面变化 & 控制力矩变化 & 角速度变化 \\
\hline $\begin{array}{c}\text { 标准控制器下不考虑参数 } \\
\text { 不确定性和外干扰 }\end{array}$ & $\begin{array}{c}40 \text { s左右达到 } 0.005 \text { 量级, } \\
\text { 后续阶段波动明显 }\end{array}$ & $\begin{array}{c}40-60 \mathrm{~s} \text { 期间达到 } \\
0.0005 \text { 量级 }\end{array}$ & $\begin{array}{c}\text { 在0-200 s阶段内存在 } \\
\text { 明显波动现象 }\end{array}$ & $\begin{array}{l}40 \text { s左右达到 } 0.1 \text { 量级, } \\
\text { 后续阶段波动明显 }\end{array}$ \\
\hline $\begin{array}{c}\text { 标准控制器下考虑参数 } \\
\text { 不确定性和外干扰 }\end{array}$ & $\begin{array}{c}50 \mathrm{~s} \text { 左右达到 } 0.01 \text { 量级, } \\
\text { 后续阶段波动较大 }\end{array}$ & $\begin{array}{c}60-70 \mathrm{~s} \text { 期间达到 } \\
0.0005 \text { 量级 }\end{array}$ & $\begin{array}{c}\text { 整个过程存在明显波动, } \\
40 \mathrm{~s} \text { 前抖振现象明显 }\end{array}$ & $\begin{array}{l}50 \mathrm{~s} \text { 左右达到 } 0.1 \text { 量级, } \\
\text { 后续阶段波动明显 }\end{array}$ \\
\hline $\begin{array}{l}\text { AMSVSC方法下考虑参数 } \\
\text { 不确定性和外干扰 }\end{array}$ & $\begin{array}{c}40 \mathrm{~s} \text { 左右已达到 } 0.002 \text { 量级, } \\
\text { 后续阶段波动明显 }\end{array}$ & $\begin{array}{c}40 \mathrm{~s} \text { 处开始已达到 } \\
0.0005 \text { 量级 }\end{array}$ & $\begin{array}{l}0-200 \mathrm{~s} \text { 阶段内存在明显 } \\
\text { 波动, } 60 \mathrm{~s} \text { 前波动比较明显 }\end{array}$ & $\begin{array}{c}40 \mathrm{~s} \text { 后能够达到 } 0.05 \text { 量级, } \\
\text { 后续阶段波动明显 }\end{array}$ \\
\hline $\begin{array}{l}\text { MCSVS与AMSVSC联合作用下 } \\
\text { 考虑参数不确定性和外干扰 }\end{array}$ & $\begin{array}{c}40 \text { s左右已达到 } 0.002 \text { 量级, } \\
\text { 后续阶段无明显波动 }\end{array}$ & $\begin{array}{c}40 \mathrm{~s} \text { 处开始已达到 } \\
0.0001 \text { 量级 }\end{array}$ & $\begin{array}{c}\text { 整个过程输出力矩变化 } \\
\text { 无明显波动 }\end{array}$ & $\begin{array}{l}40 \mathrm{~s} \text { 后能够达到 } 0.04 \text { 量级, } \\
\text { 后续阶段无明显波动 }\end{array}$ \\
\hline
\end{tabular}

为了解决传统滑模变结构控制器控制输出力矩颤振的 问题, 将自适应控制率与滑模变结构控制相结合, 提出 了改进型滑模变结构控制器, 通过Lyapunove判定定理 完成了对新控制方法稳定性的证明. 为降低姿态机动 过程引起的柔性附件残余振动，提出将MCSVS控制方 法和智能压电陶瓷相结合应用的方式. 为了证明新提
出控制策略的效果, 进行了仿真分析. 仿真结果表明, 应用新控制策略后, 控制输出力矩波动得到明显降低, 柔性附件振动显著降低, 最终输出控制力矩变化更加 平滑. 通过新控制策略的应用, 姿态机动完成后, 在考 虑参数不确定性和外干扰情况下, 系统姿态精度得到 提升.

\section{参考文献}

1 Loquen T, Plinval H D, Cumer C, et al. Attitude control of satellites with flexible appendages: A structured $H_{\infty}$ control design. In: AIAA Guidance, Navigation, and Control Conference. Minneapolis, 2012. AIAA 2012-4845

2 Liu D, Yang D, Xi J, et al. An optimal maneuver control method for the spacecraft with flexible appendages. In: Astrodynamics Conference. Minneapolis, 1988. 294-300

3 Cai X L. Dynamics and control of space rendezvous and docking (in Chinese). Dissertation for Doctoral Degree. Harbin: Harbin Institute of Technology, 1991. 39-56 [蔡晓雷. 空间交会对接动力学与控制初探. 博士学位论文. 哈尔滨: 哈尔滨工业大学, 1991. 39-56]

4 Shan J J, Liu T. Optimal control strategy for large angle maneuver of aircraft based on active vibration suppression method of component synthesis (in Chinese). Chin J Aeronaut, 2002, 23: 62-65 [陕晋军, 刘噋. 应用分力合成主动振动抑制方法的最优飞行器大角度机动控制策 略. 航空学报, 2002, 23: 62-65]

5 Shan J, Sun D, Liu D. Design for robust component synthesis vibration suppression of flexible structures with on-off actuators. IEEE Trans Robot Automat, 2004, 20: 512-525

6 Hu Q, Liu Y A. Hybrid scheme of feed-forward/feedback control for vibration suppression of flexible spacecraft with on-off actuators during attitude maneuver. Int J Inform Tech, 2005, 11: 95-107

$7 \mathrm{Hu}$ Q. Attitude maneuver and vibration control of flexible spacecraft using computed feed-forward input commands and proportional-derivative feedback. J Aerosp Eng, 2007, 221: 785-794

8 Zhang J Y, Liu T, Zheng L W. Closed-loop feedback control of spacecraft based on the component synthesis method (in Chinese). J Harbin Inst Tech, 2010, 42: 384-388 [张建英, 刘暾, 郑立伟. 基于分力合成方法的航天器闭环反馈控制. 哈尔滨工业大学学报, 2010, 42: 384-388]

9 Zhang J Y, Liu T, Zheng L W. Component synthesis active vibration suppression method of flexible spacecraft based on PWM (in Chinese). Chin J Space Sci, 2010, 30: 66-72 [张建英, 刘暾, 郑立伟. 基于PWM的挠性航天器分力合成主动振动抑制方法. 空间科学学报, 2010, 30: 66-72]

10 Qiao J, Li X, Xu J. A composite disturbance observer and $H_{\infty}$ control scheme for flexible spacecraft with measurement delay and input delay. Chin J Aeronaut, 2019, 32: 1472-1480

11 Sofyalı A, Jafarov E M, Wisniewski R. Robust and global attitude stabilization of magnetically actuated spacecraft through sliding mode. Aerosp Sci Tech, 2018, 76: 91-104

12 Liu X, Zhang M, Wang S. Adaptive region tracking control with prescribed transient performance for autonomous underwater vehicle with 
thruster fault. Ocean Eng, 2020, 196: 106804

13 Zieliński C, Stefańczyk M, Kornuta T, et al. Variable structure robot control systems: The RAPP approach. Robot Autonom Syst, 2017, 94: 226244

14 Liu C, Sun Z, Ye D, et al. Robust adaptive variable structure tracking control for spacecraft chaotic attitude motion. IEEE Access, 2018, 6: 38513857

15 Atam E, Patteeuw D, Antonov S P, et al. Optimal control approaches for analysis of energy use minimization of hybrid ground-coupled heat pump systems. IEEE Trans Contr Syst Technol, 2015, 24: 525-540

16 Abouaïssa H, Chouraqui S. On the control of robot manipulator: A model-free approach. J Comput Sci, 2019, 31: 6-16

17 Rani M, Ruchika M, Kumar N. Intelligent tracking control of redundant robot manipulators including actuator dynamics. Procedia Comput Sci, 2018, 125: 50-58

18 Wang C, Li Y, Hu Q, et al. Event-triggered adaptive control for attitude tracking of spacecraft. Chin J Aeronaut, 2019, 32: 454-462

19 Maganti G B, Singh S N. Simplified adaptive control of an orbiting flexible spacecraft. Acta Astronaut, 2007, 61: 575-589

20 Chen Z Y, Huang J. Attitude tracking and disturbance rejection of rigid spacecraft by adaptive control. IEEE Trans Automat Contr, 2009, 54: $600-605$

21 Zhu Z, Xia Y, Fu M. Adaptive sliding mode control for attitude stabilization with actuator saturation. IEEE Trans Ind Electron, 2011, 58: 48984907

22 Onoda J, Minesugi K, Oh H U. Semiactive vibration suppression with electrorheological-fluid dampers. AIAA J, 2012, 35: 1844-1852

$23 \mathrm{Li} \mathrm{L}, \mathrm{Li} \mathrm{X}, \mathrm{Hu}$ Y. Free vibration analysis of nonlocal strain gradient beams made of functionally graded material. Int J Eng Sci, 2016, 102: 77-92

24 Wang X, Pommier-Budinger V, Reysset A, et al. Simultaneous compensation of hysteresis and creep in a single piezoelectric actuator by openloop control for quasi-static space active optics applications. Control Eng Practice, 2014, 33: 48-62

25 Azadi M, Fazelzadeh S A, Eghtesad M, et al. Vibration suppression and adaptive-robust control of a smart flexible satellite with three axes maneuvering. Acta Astronaut, 2011, 69: 307-322

26 Onoda J, Makihara K, Minesugi K. Energy-recycling semi-active method for vibration suppression with piezoelectric transducers. AIAA J, 2012, 41: 711-719

27 Di Gennaro S. Output stabilization of flexible spacecraft with active vibration suppression. IEEE Trans Aerosp Electron Syst, 2003, 39: 747-759 


\title{
Flexible spacecraft attitude maneuver via adaptive sliding mode control and mixed component synthesis vibration suppression
}

\author{
DONG Chao, XU ShiJie", WANG Hao, ZHANG MingXing \& XUE Pu \\ Beijing Institute of Space Long March Vehicle, Beijing 100076, China
}

\begin{abstract}
A mixed component synthesis vibration suppression method (MCSVS) is proposed to solve the problem of residual vibration of flexible attachments, which is difficult to control effectively. Theorems and inferences are provided, and their proofs are performed. Aiming at the problem that the attitude controllers cannot suppress the vibration of flexible attachments in spacecraft attitude maneuver, a strategy combining the MCSVS method with an attitude control method is proposed. Considering the existence of moment of inertia variation and external disturbance of spacecraft, an adaptive sliding mode variable structure controller is proposed. By introducing a sliding mode boundary layer and the update rate of the torque parameter, the disadvantages of torque chattering and parameter dependence are eliminated. Finally, the joint application of the MCSVS method and the adaptive variable structure controller is realized with the assistance of piezoelectric intelligent materials. Simulation results show that the control strategy without the MCSVS method cannot suppress the vibration of flexible attachments. After combining the MCSVS method with the attitude control method, the residual vibration is well controlled, and the attitude accuracy is improved.
\end{abstract}

flexible spacecraft, mixed component synthesis vibration suppression (MCSVS) method, active vibration suppression-adaptive sliding mode control

PACS: $45.05 .+\mathrm{x}, 45.82 .+\mathrm{r}, 46.40 .-\mathrm{f}$

doi: $10.1360 /$ SSPMA-2021-0116 